\title{
Dithienogermole-Based Nonfullerene Acceptors: Roles of the Side-Chains' Direction and Development of Green-Tinted Efficient Semitransparent Organic Solar Cells
}

Yongjoon Cho, † Tack Ho Lee, + Seonghun Jeong, Song Yi Park, Byongkyu Lee, Jin Young Kim,* and Changduk Yang*

Department of Energy Engineering, School of Energy and Chemical Engineering, Perovtronics Research Center, Low Dimensional Carbon Materials Center, Ulsan National Institute of Science and Technology (UNIST), 50 UNIST-gil, Ulju-gun, Ulsan 44919, Republic of Korea.

*Corresponding Authors

Jin Young Kim

E-mail: jykim@unist.ac.kr

Changduk Yang

E-mail: yang@unist.ac.kr

KEYWORDS: constitutional isomers, dithienogermole, narrow-bandgap nonfullerene acceptors, semitransparent organic solar cells, $\mathrm{Ag} / \mathrm{Sb}_{2} \mathrm{O}_{3} / \mathrm{Ag}$ electrode 


\section{Experimental Section}

Materials and characterization: All starting materials were purchased from Sigma-Aldrich and Acros and used without further purification, and PTB7-Th was purchased from Solarmer. All solvents were of ACS grade unless otherwise noted. 2-bromo-3-(2-ethylhexyl)thiophene (1), 3-(2-ethylhexyl)thiophene-2-carbaldehyde (2), and 4,4-bis(2-ethylhexyl)-2,6bis(trimethylstannyl)-4H-germolo[3,2-b:4,5-b']dithiophene (3) were synthesized according to the previously reported methods. ${ }^{1-3}{ }^{1} \mathrm{H}$ NMR and ${ }^{13} \mathrm{C}$ NMR spectra were recorded on a VNMRS $400 \mathrm{MHz}$ spectrophotometer using deutrated chloroform $\left(\mathrm{CDCl}_{3}\right)$ as solvent and tetramethylsilane (TMS) as the internal standard. HRMS spectra were measured using AccuTOF 4G+ DART. UV-Vis-NIR spectra and transmittance were recorded on a UV-1800 (SHIMADZU) spectrophotometer. For UV-vis-NIR spectra of the DTG-based acceptors, their solutions (4.00 $\mathrm{mg} \mathrm{mL}^{-1}$ in $\mathrm{CF}$ ) were used and the films were prepared by deposition from the $8.00 \mathrm{mg} \mathrm{mL}^{-1}$ concentrated CF solution via the spin-casting method at $1000 \mathrm{rpm}$. The optical bandgaps were estimated from the absorption onset of the as-cast thin films. CV measurements were performed on a Solartron electrochemical station (METEK, Versa STAT3) with a threeelectrode cell in a $0.1 \mathrm{M} \mathrm{n}$-Bu $\mathrm{NPF}_{6}$ solution in $\mathrm{CH}_{3} \mathrm{CN}$ at a scan rate of $100 \mathrm{mV} \mathrm{s}^{-1}$ at room temperature. $\mathrm{An} \mathrm{Ag} / \mathrm{Ag}^{+}\left(0.01 \mathrm{M} \mathrm{AgNO}_{3}\right.$ in $\left.\mathrm{CH}_{3} \mathrm{CN}\right)$ electrode, a platinum wire, and a materialcoated platinum electrode were used as the reference electrode, the counter electrode, and the working electrode, respectively. The $\mathrm{Ag} / \mathrm{Ag}^{+}$reference electrode was calibrated using a $\mathrm{Fc} / \mathrm{Fc}^{+}$ redox couple as an internal standard, whose oxidation potential is set at $-4.8 \mathrm{eV}$ with respect to the zero vacuum level. HOMO and LUMO energy levels were determined according to the equations $\left(E_{\mathrm{HOMO}}=-\left(E_{\mathrm{ox}}{ }^{\text {onset }}-E_{\mathrm{Fc}}{ }^{\text {onset }}+4.8\right)(\mathrm{eV})\right)$ and $\left(E_{\mathrm{LUMO}}=-\left(E_{\mathrm{red}}{ }^{\text {onset }}-E_{\mathrm{Fc}}{ }^{\text {onset }}+4.8\right)\right.$ $(\mathrm{eV})$ ). The DFT calculations were performed using the Gaussian 09 package at B3LYP 
function $\left(6-31 \mathrm{G}^{*}\right.$ and $6-31 \mathrm{G}+* *$ basis set) to elucidate the HOMO and LUMO energy levels, net dipole moments, and rotation energies after optimizing the geometry of materials.

For morphological characterization, the optimized device fabrication processing condition were used to prepare thin films and all the characterizations otherwise mentioned were performed in ambient atmospheric condition. Glass substrate was used to fabricate samples for the absorption, optical fluorescence microscopy, and thickness measurement while for all other characterizations, samples were fabricated on a silicon substrate. AFM $(1.5 \mu \mathrm{m} \times 1.5 \mu \mathrm{m})$ was performed using a Bruker Dimension Icon Atomic Force Microscope with an RTESP-150 probe in the standard tapping mode. The thickness of the films was measured using a stylus profilometer of P6, KLA Tencor. TEM analysis was performed using a JEOL USA JEM-2100F (Cs corrector) transmission electron microscope equipped with an energy dispersive X-ray analysis at an acceleration voltage of $200 \mathrm{kV}$. GIWAXS measurements were conducted at PLSII 6D and 9A U-SAXS beamline in Pohang Accelerator Laboratory (South Korea). The X-rays coming from the in-vacuum undulator (IVU) were monochromated at $11.05 \mathrm{keV}$ (wavelength of $1.12199 \AA)$. The incidence angle $\left(\sim 0.12-14^{\circ}\right)$ of X-ray beam was chosen to allow for complete penetration into the film. The GIWAXS patterns were recorded with a $2 \mathrm{D}$ CCD detector.

Synthesis of 5-bromo-4-(2-ethylhexyl)thiophene-2-carbaldehyde (1a): To a two-neck roundbottom flask, 1 (4 g, $14.5 \mathrm{mmol})$ was dissolved in $100 \mathrm{ml}$ of tetrahydrofuran under inert atmosphere. The reaction mixture was cooled in a dry ice-acetone bath at $-78{ }^{\circ} \mathrm{C}$, and lithium diisopropylamide ( $2 \mathrm{M}$ in hexane, $8.7 \mathrm{ml}, 17.4 \mathrm{mmol}$ ) was added to the solution slowly. With stirring for $1 \mathrm{~h}$, anhydrous dimethylformamide $(3.4 \mathrm{ml}, 43.9 \mathrm{mmol})$ was added and warmed to room temperature (RT). The reaction mixture was refluxed overnight with vigorous stirring. Then the mixture quenched by water was extracted with diethyl ether. The organic layer was collected and dried with anhydrous magnesium sulfate, and then the volatiles were removed 
under reduced pressure. The resulting crude mixture was purified by column chromatography using hexane/dichloromethane (DCM) as an eluent, obtaining a colorless oil $(3.61 \mathrm{~g}, 83 \%) .{ }^{1} \mathrm{H}$ $\operatorname{NMR}\left(\mathrm{CDCl}_{3}, 400 \mathrm{MHz}\right) \delta$ ppm $9.76(\mathrm{~s}, 1 \mathrm{H}), 7.42(\mathrm{~s}, 1 \mathrm{H}), 2.53(\mathrm{~d}, J=7.2 \mathrm{~Hz}, 2 \mathrm{H}), 1.66-1.59$ (m, 1H), 1.36-1.21 (m, 8H), 0.93-0.85 (m, 6H). HRMS (ESI) $m / z 302.0412\left(\mathrm{C}_{13} \mathrm{H}_{19} \mathrm{BrOS}\right.$ calcd. for $m / z$ 302.0340).

Synthesis of 5-bromo-3-(2-ethylhexyl)thiophene-2-carbaldehyde (2a): To a two-neck roundbottom flask, 2 (4 g, $17.8 \mathrm{mmol})$ was dissolved in $200 \mathrm{ml}$ of $\mathrm{CF}$, then bromine $(0.91 \mathrm{ml}, 17.8$ mmol) was slowly added at RT. Upon completion of the addition, the reaction mixture was stirred for $10 \mathrm{~h}$. Then the mixture quenched by sodium carbonate solution was extracted with CF. The organic layer was collected and dried with anhydrous magnesium sulfate, and then the volatiles were removed under reduced pressure. The resulting crude mixture was purified by column chromatography using hexane/dichloromethane (DCM) as an eluent, obtaining a colorless oil (4.97 g, 92\%). ${ }^{1} \mathrm{H}$ NMR $\left(\mathrm{CDCl}_{3}, 400 \mathrm{MHz}\right) \delta \mathrm{ppm} 9.89$ (s, 1H), 6.96 (s, 1H), 2.82 $(\mathrm{d}, J=7.2 \mathrm{H}, 2 \mathrm{H}), 1.65-1.55(\mathrm{~m}, 1 \mathrm{H}), 1.41-1.21(\mathrm{~m}, 8 \mathrm{H}), 0.96-0.83(\mathrm{~m}, 6 \mathrm{H})$. HRMS (ESI) $m / z$ $302.0389\left(\mathrm{C}_{13} \mathrm{H}_{19} \mathrm{BrOS}\right.$ calcd. for $m / z$ 302.0340).

Synthesis of 5,5'-(4,4-bis(2-ethylhexyl)-4H-germolo[3,2-b:4,5-b']dithiophene-2,6diyl)bis(4-(2-ethylhexyl)thiophene-2-carbaldehyde) (4): To a two-neck round-bottom flask of 3 (1 g, $1.27 \mathrm{mmol})$, 1a (0.96 g, $3.18 \mathrm{mmol})$, and tetrakis(triphenylphosphine)palladium(0) $(0.073 \mathrm{~g}, 0.063 \mathrm{mmol})$ in toluene $(20 \mathrm{ml})$ were added under inert atmosphere. The reaction mixture was refluxed overnight with vigorous stirring. After cooling to room temperature, the volatile was removed under reduced pressure. The resulting crude mixture was purified by column chromatography using hexane/dichloromethane (DCM) as an eluent, obtaining a red solid (0.88 g, 76\%). ${ }^{1} \mathrm{H} \mathrm{NMR}\left(\mathrm{CDCl}_{3}, 400 \mathrm{MHz}\right) \delta \mathrm{ppm} 9.83(\mathrm{~s}, 2 \mathrm{H}), 7.56(\mathrm{~s}, 2 \mathrm{H}), 7.26(\mathrm{~s}, 2 \mathrm{H})$, $2.77(\mathrm{~d}, J=7.2 \mathrm{~Hz}, 4 \mathrm{H}), 1.66-1.60(\mathrm{~m}, 2 \mathrm{H}), 1.55-1.48(\mathrm{~m}, 2 \mathrm{H}), 1.41-1.11(\mathrm{~m}, 34 \mathrm{H}), 0.98-0.77$ (m, 26H). HRMS (ESI) $m / z, 908.3819\left(\mathrm{C}_{50} \mathrm{H}_{74} \mathrm{GeO}_{2} \mathrm{~S}_{4}\right.$ calcd. for $m / z$ 908.3783). 
Synthesis of 5,5'-(4,4-bis(2-ethylhexyl)-4H-germolo[3,2-b:4,5-b']dithiophene-2,6diyl)bis(3-(2-ethylhexyl)thiophene-2-carbaldehyde) (5): 5 was synthesized through the same synthetic route as $\mathbf{4}$, and $\mathbf{2 a}$ was used instead of $\mathbf{1 a}$, yielding a red solid $(0.82 \mathrm{~g}, 71 \%) .{ }^{1} \mathrm{H}$ NMR $\left(\mathrm{CDCl}_{3}, 400 \mathrm{MHz}\right) \delta \mathrm{ppm} 9.97(\mathrm{~s}, 2 \mathrm{H}), 7.30(\mathrm{~s}, 2 \mathrm{H}), 7.01(\mathrm{~s}, 2 \mathrm{H}), 2.85(\mathrm{~d}, J=7.2 \mathrm{~Hz}, 4 \mathrm{H})$, 1.68-1.62 (m, 2H), 1.53-1.42 (m, 2H), 1.42-1.11 (m, 34H), 0.95-0.77 (26H). HRMS (ESI) m/z $908.3844\left(\mathrm{C}_{50} \mathrm{H}_{74} \mathrm{GeO}_{2} \mathrm{~S}_{4}\right.$ calcd. for $m / z$ 908.3783).

Synthesis of DTG-IW: To a two-neck round-bottom flask of $\mathbf{1 a}(0.5 \mathrm{~g}, 0.55 \mathrm{mmol})$ and $2 \mathrm{FIC}$ $(0.45 \mathrm{~g}, 1.96 \mathrm{mmol})$ in $\mathrm{CF}(30 \mathrm{ml})$ with pyridine $(0.5 \mathrm{ml})$ were added. The reaction mixture was stirred at $80{ }^{\circ} \mathrm{C}$ overnight. After cooling to room temperature, the mixture quenched by water was extracted with DCM. After dried over anhydrous magnesium sulfate and the volatile was removed under reduced pressure. The residue was purified by column chromatography using hexane/DCM as an eluent, yielding a dark blue solid $(0.67 \mathrm{~g}, 91 \%) .{ }^{1} \mathrm{H}$ NMR $\left(\mathrm{CDCl}_{3}\right.$, $400 \mathrm{MHz}) \delta$ ppm 8.78 (s, 2H), $8.54(\mathrm{dd}, J=10.0 \mathrm{~Hz}, 6.5 \mathrm{~Hz}, 2 \mathrm{H}), 7.68$ (m, 2H), 7.65 (s, 2H), $7.57(\mathrm{~s}, 2 \mathrm{H}), 2.83(\mathrm{~d}, J=7.3 \mathrm{~Hz}, 4 \mathrm{H}), 1.78(\mathrm{~m}, 2 \mathrm{H}), 1.55-1.49(\mathrm{~m}, 4 \mathrm{H}), 1.47-1.11(\mathrm{~m}, 32 \mathrm{H})$, 0.95-0.87 (m, 14H), 0.87-0.80 (m, 12H). ${ }^{13} \mathrm{C} \mathrm{NMR}\left(\mathrm{CDCl}_{3}, 100 \mathrm{MHz}\right) \delta \mathrm{ppm} 186.00,158.29$, $153.22,153.07,150.55,149.51,149.45,149.42,148.15,148.00,140.26,137.41,137.12,136.59$, $134.48,134.10,132.07,121.33,115.08,114.87,114.28,114.20,112.69,112.50,69.72,39.53$, $37.00,35.53,33.81,32.57,28.95,28.79,28.68,25.77,23.06,23.04,21.00,14.16,14.14,10.88$, 10.68, 10.66. HRMS (ESI) $m / z 1332.4221\left(\mathrm{C}_{74} \mathrm{H}_{78} \mathrm{~F}_{4} \mathrm{GeN}_{4} \mathrm{O}_{2} \mathrm{~S}_{4}\right.$ calcd. for $m / z$ 1332.4156).

Synthesis of DTG-OW: DTG-OW was synthesized through the same synthetic route as DTGIW, and 5 was used instead of $\mathbf{4}$, yielding a dark blue solid $(0.64 \mathrm{~g}, 92 \%) .{ }^{1} \mathrm{H} \mathrm{NMR}\left(\mathrm{CDCl}_{3}\right.$, $400 \mathrm{MHz}) \delta$ ppm 8.95 (s, 2H), $8.54(\mathrm{dd}, J=10.0 \mathrm{~Hz}, 6.5 \mathrm{~Hz}, 2 \mathrm{H}), 7.67$ (m, 2H), $7.60(\mathrm{~s}, 2 \mathrm{H})$, $7.20(\mathrm{~s}, 2 \mathrm{H}), 2.90(\mathrm{~d}, J=7.3 \mathrm{~Hz}, 4 \mathrm{H}), 1.71(\mathrm{~m}, 2 \mathrm{H}), 1.55-1.49(\mathrm{~m}, 4 \mathrm{H}), 1.44-1.13(\mathrm{~m}, 32 \mathrm{H})$, 0.98-0.80 (m, 26H). ${ }^{13} \mathrm{C} \mathrm{NMR}\left(\mathrm{CDCl}_{3}, 100 \mathrm{MHz}\right) \delta \mathrm{ppm} 186.04,162.44,159.16,159.13$, $153.09,151.95,149.35,149.34,149.27,149.22,138.61,136.55,136.52,134.56,131.97$, 
$131.09,127.24,119.79,115.00,114.94,114.79,114.57,112.52,112.33,68.72,41.88,36.98$, $35.52,34.60,32.14,28.90,28.85,28.65,25.49,23.05,23.02,21.01,14.16,14.13,10.89,10.80$, 10.73. HRMS (ESI) $m / z$ 1332.4214 $\left(\mathrm{C}_{74} \mathrm{H}_{78} \mathrm{~F}_{4} \mathrm{GeN}_{4} \mathrm{O}_{2} \mathrm{~S}_{4}\right.$ calcd. for $m / z$ 1332.4156).

OSC fabrication \& characterization: Patterned ITO glasses were cleaned with distilled water, acetone, and isopropanol, in sequence. Poly(3,4-ethylenedioxythiophene): polystyrene sulfonic acid (PEDOT:PSS) was spin-coated onto the ITO substrate and annealed at $150{ }^{\circ} \mathrm{C}$ for 10 min. To prepare active layers, PTB7-Th was blended with the acceptors in a chlorobenzene:1-chloronaphthalene ( $\mathrm{CB}: \mathrm{CN}=99: 1$ in volume ratio). On top of the PEDOT:PSS layer, active layers were spin-casted in a $\mathrm{N}_{2}$ filled glove box. Next, $\mathrm{ZnO}$ nanoparticles dispersed in methanol were spin-casted on top of the active layer. The device was pumped down under vacuum $\left(<10^{-6}\right.$ Torr $)$, and $\mathrm{Al}(100 \mathrm{~nm})$ was deposited by thermal evaporation. To fabricate green-tinted semitransparent OSCs, silver slug (Alfa Aesar, 99.999\% metals basis) and antimony (III) oxide (Aldrich, 99.999\% metals basis) were thermally evaporated to deposit $\mathrm{Ag}(25 \mathrm{~nm}) / \mathrm{Sb}_{2} \mathrm{O}_{3}(65 \mathrm{~nm}) / \mathrm{Ag}(25 \mathrm{~nm})$ under vacuum condition $\left(<10^{-6}\right.$ Torr). The area of the $\mathrm{Al}$ electrode defines an active area of the device as $4.0 \mathrm{~mm}^{2}$. Measurements were carried out inside a glove box by using a high quality optical fiber to guide the light from a solar simulator equipped with a Keithley 2635A source measurement unit. A mask $\left(4.0 \mathrm{~mm}^{2}\right)$ made of a thin metal was attached to each cell for the photovoltaic characteristic measurements under AM $1.5 \mathrm{G}$ illumination at $100 \mathrm{~mW} \mathrm{~cm} \mathrm{~cm}^{-2}$. The EQE measurements were performed using a PV measurement QE system using monochromatic light from a xenon lamp under ambient conditions. The monochromatic light was chopped at 100 $\mathrm{Hz}$ and the intensity was calibrated relative to a standard $\mathrm{Si}$ photodiode using a lock-inamplifier. The SCLC method was carried by fabricating the hole-only (ITO/PEDOT:PSS/active layer/MoO $3 / \mathrm{Ag}$ ) and electron-only (FTO/active layer/Al, FTO: 
fluorine-doped tin oxide) diodes. Changes in electrical potential due the built-in potential $\left(V_{\mathrm{bi}}\right)$ were accounted for to ensure accuracy in the measurements.

(a)

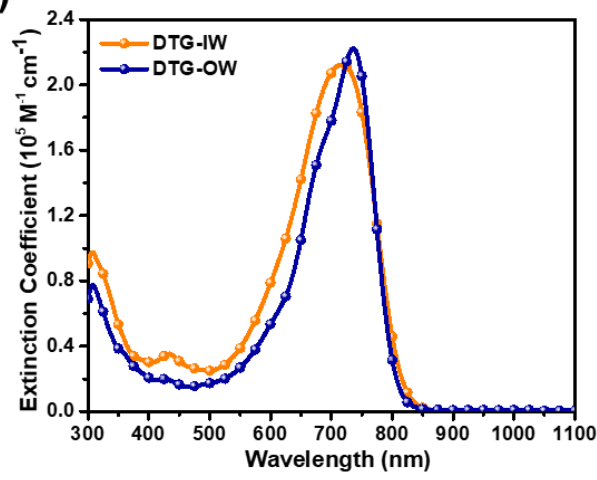

(b)

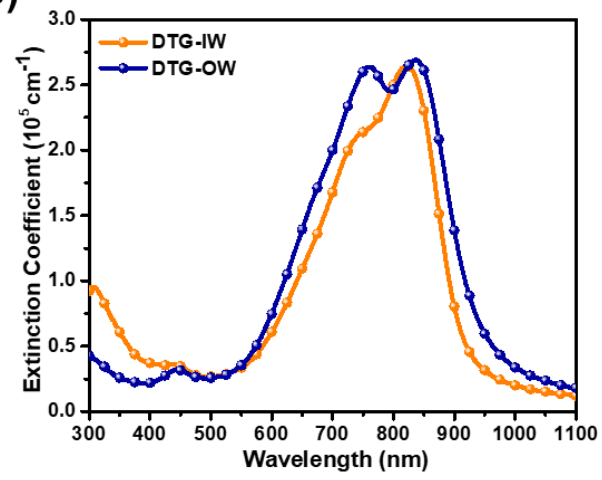

Figure S1. Extinction coefficient of DTG-IW and DTG-OW in (a) solution and (b) thin film.
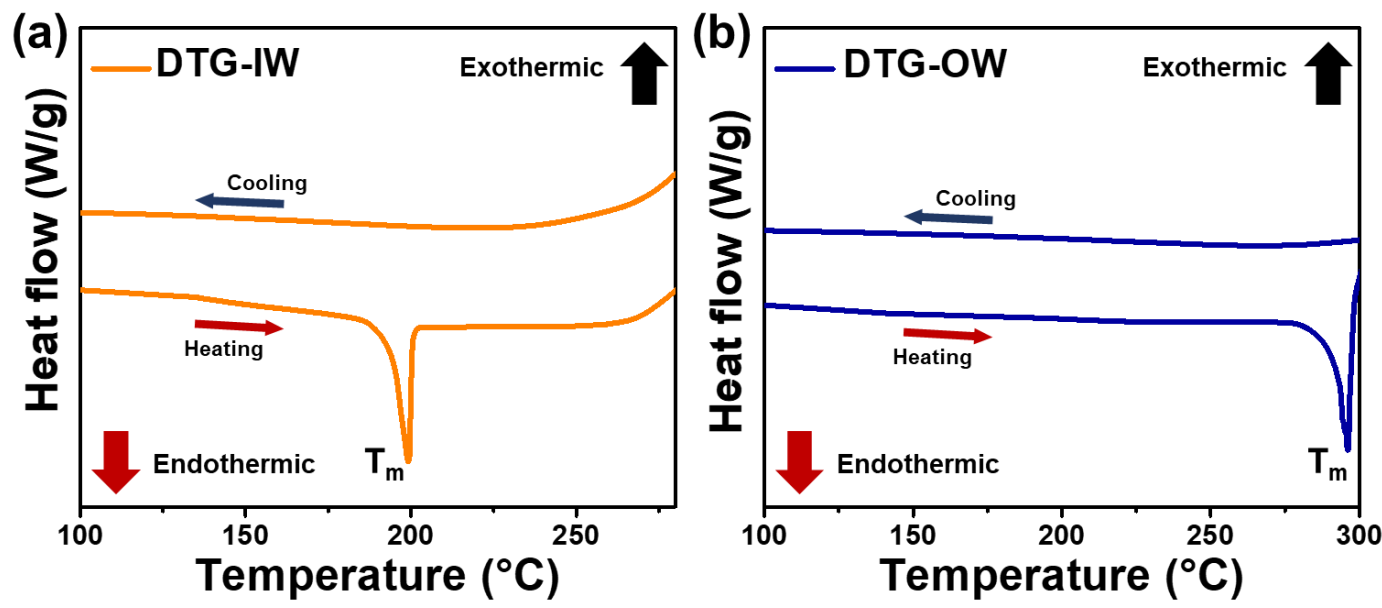

Figure S2. DSC thermogram of (a) DTG-IW and (b) DTG-OW in $\mathrm{N}_{2}$ atmosphere at a scan rate of $10{ }^{\circ} \mathrm{C} \mathrm{min}{ }^{-1}$.

Table S1. DSC thermal parameters of DTG-IW and DTG-OW.

\begin{tabular}{lcc}
\hline & $\mathrm{T}_{\mathrm{m}}$ & $\begin{array}{l}\Delta \mathrm{H}_{\mathrm{m}} \\
{\left[{ }^{\circ} \mathrm{C}\right]}\end{array}$ \\
\hline DTG-IW & 199.2 & 11.27 \\
DTG-OW & 296.2 & 33.16 \\
\hline
\end{tabular}


Side

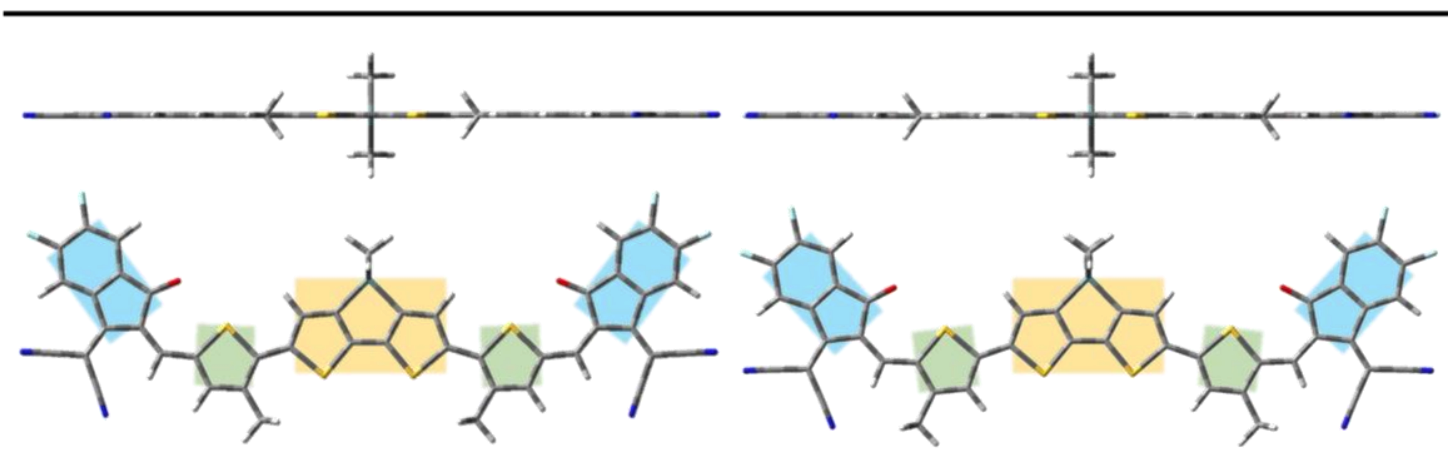

Figure S3. Optimized geometries of DTG-IW and DTG-OW.

Table S2. Calculated dipole moments and energy levels of the DTG-IW and DTG-OW.

\begin{tabular}{|c|c|c|c|c|c|c|}
\hline & \multicolumn{3}{|c|}{ Vector } & \multirow{2}{*}{$\begin{array}{l}\text { Dipole Moment } \\
\text { [D] }\end{array}$} & \multirow{2}{*}{$\begin{array}{c}\mathrm{E}_{\mathrm{HOMO}}{ }^{\mathrm{DFT}} \\
{[\mathrm{eV}]}\end{array}$} & \multirow{2}{*}{$\begin{array}{c}\mathrm{E}_{\text {LUMO }}{ }^{\mathrm{DFT}} \\
{[\mathrm{eV}]}\end{array}$} \\
\hline & $x$ & $y$ & z & & & \\
\hline DTG-IW & -0.0004 & 0.8366 & -0.0010 & 0.8366 & -5.60 & -3.80 \\
\hline DTG-OW & -0.0004 & 1.7629 & -0.0437 & 1.7634 & -5.66 & -3.80 \\
\hline
\end{tabular}

Table S3. Photovoltaic parameters of the DTG-IW:PTB7-Th and DTG-OW:PTB7-Th devices with various conditions.

\begin{tabular}{|c|c|c|c|c|c|c|}
\hline Acceptor & $A: D$ ratio & Solvent & $\begin{array}{c}J_{\mathrm{SC}} \\
{\left[\mathrm{mA} \mathrm{cm}^{-2}\right]}\end{array}$ & $\begin{array}{l}V_{\mathrm{OC}} \\
{[\mathrm{V}]}\end{array}$ & $F F$ & $\begin{array}{l}\text { PCE } \\
{[\%]} \\
\end{array}$ \\
\hline \multirow{9}{*}{ DTG-IW:PTB7-Th } & $1: 1$ & CB: $1 \% \mathrm{CN}$ & 14.5 & 0.719 & 0.546 & 5.67 \\
\hline & $1.25: 1$ & CB: $1 \% \mathrm{CN}$ & 18.8 & 0.723 & 0.590 & 8.02 \\
\hline & \multirow{6}{*}{$1.5: 1$} & CB: $1 \% \mathrm{CN}$ & 19.2 & 0.719 & 0.662 & 9.16 \\
\hline & & $\mathrm{CB}: 2 \% \mathrm{CN}$ & 17.1 & 0.726 & 0.661 & 8.22 \\
\hline & & CB:0.5\%DPE & 19.3 & 0.724 & 0.559 & 7.82 \\
\hline & & CB:1\%DPE & 19.7 & 0.724 & 0.605 & 8.63 \\
\hline & & CB:1\%DIO & 12.8 & 0.721 & 0.448 & 4.13 \\
\hline & & CF:2\%CN & 13.1 & 0.695 & 0.393 & 3.59 \\
\hline & $2: 1$ & $\mathrm{CB}: 1 \% \mathrm{CN}$ & 17.5 & 0.719 & 0.642 & 8.08 \\
\hline \multirow{6}{*}{ DTG-OW:PTB7-Th } & $1: 1$ & $\mathrm{CB}: 1 \% \mathrm{CN}$ & 13.3 & 0.693 & 0.450 & 4.16 \\
\hline & $1.25: 1$ & CB: $1 \% \mathrm{CN}$ & 15.5 & 0.694 & 0.508 & 5.45 \\
\hline & \multirow{3}{*}{$1.5: 1$} & CB: $1 \% \mathrm{CN}$ & 19.1 & 0.693 & 0.507 & 6.71 \\
\hline & & CB: $2 \% \mathrm{CN}$ & 16.7 & 0.673 & 0.537 & 6.04 \\
\hline & & CB:0.5\%DPE & 13.7 & 0.698 & 0.576 & 5.49 \\
\hline & $2: 1$ & $\mathrm{CB}: 1 \% \mathrm{CN}$ & 16.2 & 0.692 & 0.663 & 7.45 \\
\hline
\end{tabular}




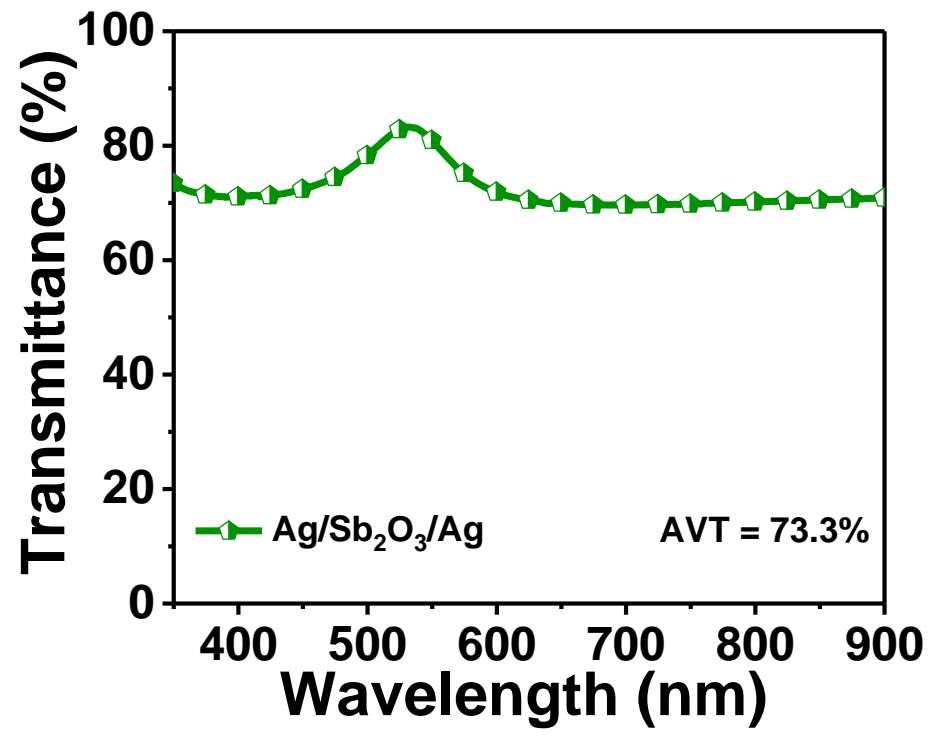

Figure S4. Transmittance spectra of $\mathrm{Ag} / \mathrm{Sb}_{2} \mathrm{O}_{3} / \mathrm{Ag}$ electrode.
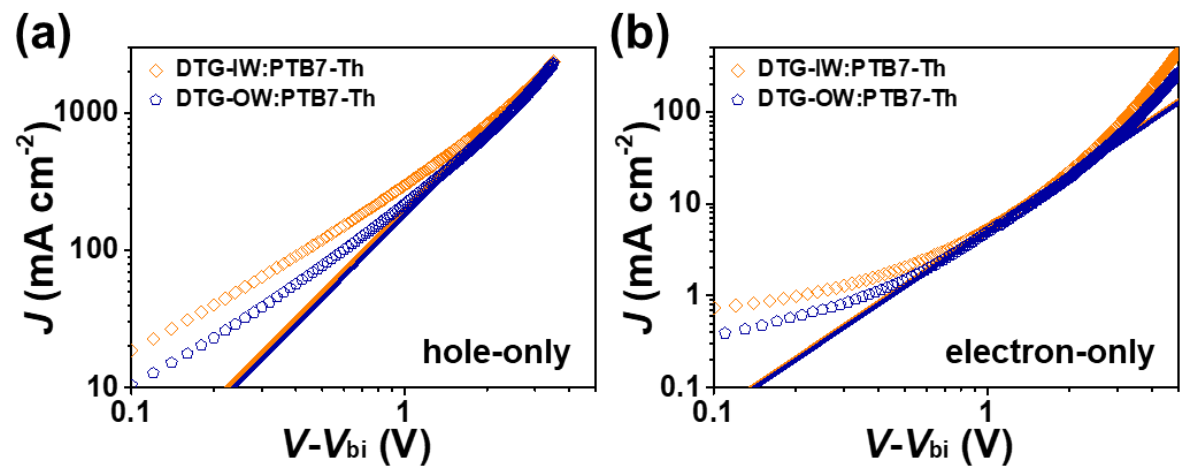

Figure S5. SCLC plots of the optimized DTG-IW:PTB7-Th and DTG-OW:PTB7-Th devices.
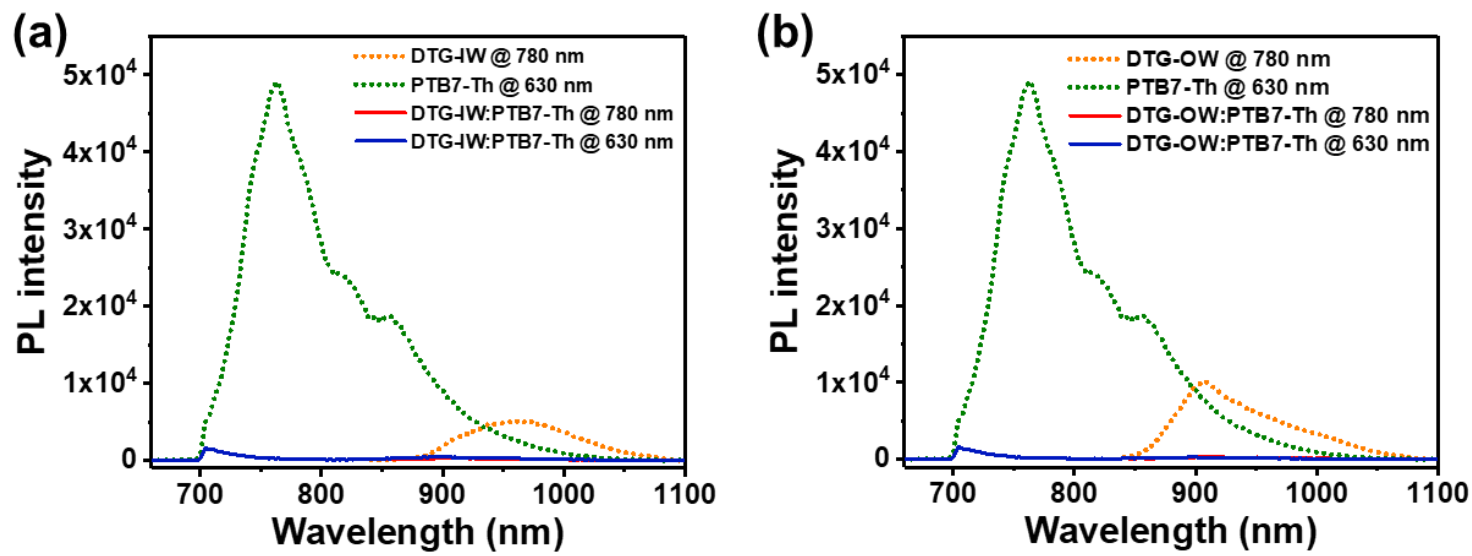

Figure S6. PL spectra of (a) DTG-IW, PTB7-Th, and DTG-IW:PTB7-Th films, and (b) DTG-OW, PTB7-Th, and DTG-OW:PTB7-Th films. 

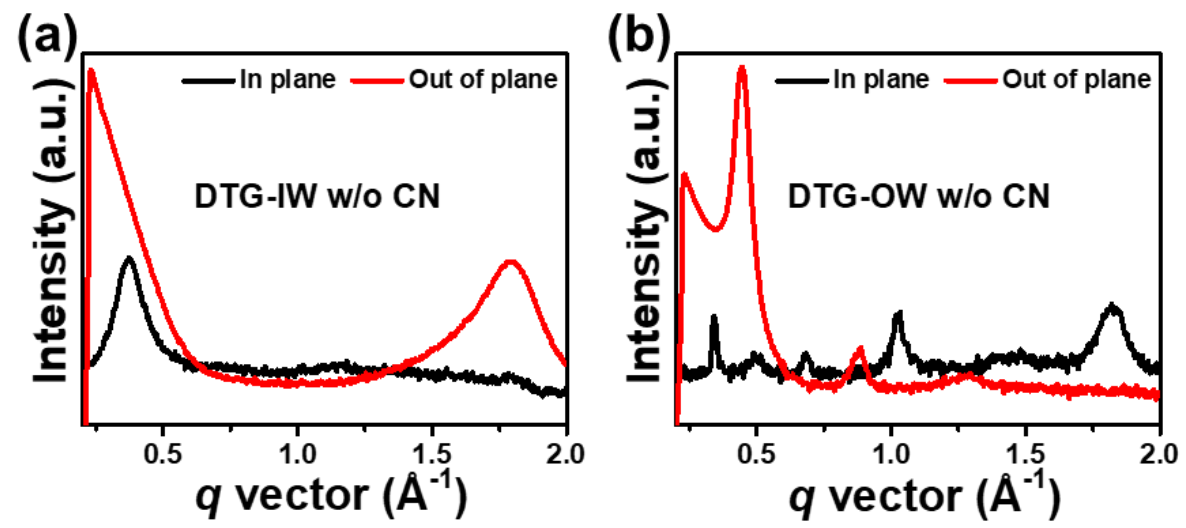

Figure S7. Line cut profiles of (a) DTG-IW (b) DTG-OW films without CN additive.

Table S4. GIWAXD parameters of neat DTG-IW, DTG-OW and PTB7-Th films and optimized blend films of DTG-IW:PTB7-Th and DTG-OW:PTB7-Th without or with $1 \% \mathrm{CN}$ additive.

\begin{tabular}{|c|c|c|c|c|c|c|c|c|c|c|c|c|}
\hline & \multicolumn{6}{|c|}{ Out-of-Plane } & \multicolumn{6}{|c|}{ In-Plane } \\
\hline & \multicolumn{3}{|c|}{$\pi-\pi$ stacking cell axis $(010)$} & \multicolumn{3}{|c|}{ lamellar stacking cell axis (100) } & \multicolumn{3}{|c|}{$\pi-\pi$ stacking cell axis $(010)$} & \multicolumn{3}{|c|}{ lamellar stacking cell axis (100) } \\
\hline & $\begin{array}{c}\mathrm{q} \\
{\left[\AA^{-1}\right]}\end{array}$ & $\begin{array}{c}\mathrm{d}- \\
\text { spacing } \\
{[\AA]}\end{array}$ & $\begin{array}{l}\text { Coherence } \\
\text { length }[\AA]\end{array}$ & $\begin{array}{c}\mathrm{q} \\
{\left[\AA^{-1}\right]}\end{array}$ & $\begin{array}{c}\mathrm{d}- \\
\text { spacing } \\
{[\AA]}\end{array}$ & $\begin{array}{c}\text { Coherence } \\
\text { length } \\
{[\AA]}\end{array}$ & $\begin{array}{c}\mathrm{q} \\
{\left[\AA^{-1}\right]}\end{array}$ & $\begin{array}{c}\mathrm{d}- \\
\text { spacing } \\
{[\AA]}\end{array}$ & $\begin{array}{l}\text { Coherence } \\
\text { length }[\AA]\end{array}$ & $\begin{array}{c}\mathrm{q} \\
{\left[\AA^{-1}\right]}\end{array}$ & $\begin{array}{c}\mathrm{d}- \\
\text { spacing } \\
{[\AA]}\end{array}$ & $\begin{array}{c}\text { Coherence } \\
\text { length } \\
{[\AA]}\end{array}$ \\
\hline $\begin{array}{l}\text { DTG TI-4FIC } \\
\text { w/o CN }\end{array}$ & 1.783 & 3.524 & 30.309 & & $\mathrm{~N} / \mathrm{A}$ & & & $\mathrm{N} / \mathrm{A}$ & & 0.374 & 16.784 & 58.955 \\
\hline $\begin{array}{l}\text { DTG TO- } \\
\text { 4FIC } \\
\text { w/o CN }\end{array}$ & & N/A & & 0.444 & 14.147 & 168.932 & 1.820 & 3.452 & 58.696 & & $\mathrm{~N} / \mathrm{A}$ & \\
\hline $\begin{array}{l}\text { PTB7-Th } \\
\text { w/o CN }\end{array}$ & 1.605 & 3.915 & 17.358 & & $\mathrm{~N} / \mathrm{A}$ & & & N/A & & 0.262 & 23.950 & 51.440 \\
\hline $\begin{array}{l}\text { DTG TI- } \\
\text { 4FIC:PTB7- } \\
\text { Th } \\
\text { w/o CN }\end{array}$ & 1.746 & 3.599 & 32.003 & & $\mathrm{~N} / \mathrm{A}$ & & & $\mathrm{N} / \mathrm{A}$ & & 0.315 & 19.948 & 47.410 \\
\hline $\begin{array}{l}\text { DTG TO- } \\
\text { 4FIC:PTB7- } \\
\text { Th } \\
\text { w/o CN }\end{array}$ & 1.674 & 3.754 & 22.790 & 0.415 & 15.134 & 79.375 & 1.831 & 3.432 & 59.075 & 0.309 & 20.308 & 43.304 \\
\hline $\begin{array}{l}\text { DTG TI- } \\
\text { 4FIC:PTB7- } \\
\text { Th } \\
\text { w/ } 1 \% \mathrm{CN}\end{array}$ & 1.753 & 3.585 & 32.078 & 0.476 & 13.207 & 129.992 & 1.715 & 3.664 & 12.316 & 0.285 & 22.015 & 67.558 \\
\hline $\begin{array}{l}\text { DTG TO- } \\
\text { 4FIC:PTB7- } \\
\text { Th } \\
\text { w/ } 1 \% \mathrm{CN}\end{array}$ & 1.730 & 3.631 & 25.627 & 0.433 & 14.511 & 89.216 & 1.832 & 3.431 & 60.433 & 0.278 & 22.581 & 60.310 \\
\hline
\end{tabular}


Table S5. GIWAXD circular line cut ratio of (010) $\pi-\pi$ stacking for neat DTG-IW and DTGOW films without $\mathrm{CN}$ and their optimized blend films with $1 \% \mathrm{CN}$.

\begin{tabular}{lcc}
\hline & Face on & $\begin{array}{c}\text { Edge on } \\
{[\%]}\end{array}$ \\
\hline DTG TI-4FIC & 100 & 0 \\
DTG TO-4FIC & 0 & 100 \\
DTG TI-4FIC:PTB7-Th & 100 & 0 \\
DTG TO-4FIC:PTB7-Th & 33.7 & 66.3 \\
\hline
\end{tabular}
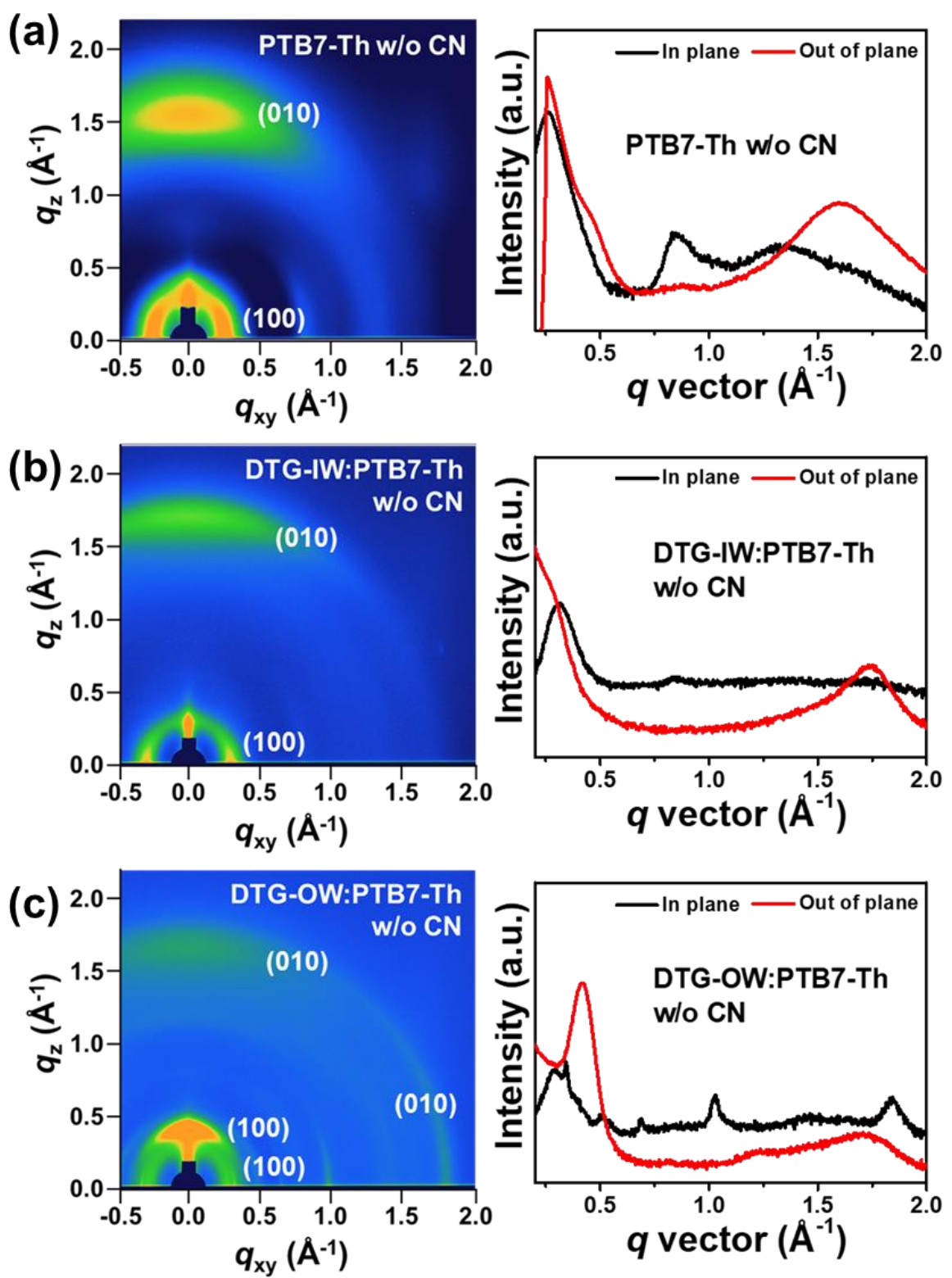

Figure S8. 2D GIWAXD pictures and line cut profiles of the (a) neat PTB7-Th film, and blend films of (b) DTG-IW:PTB7-Th and (c) DTG-OW:PTB7-Th without CN. 
(a)

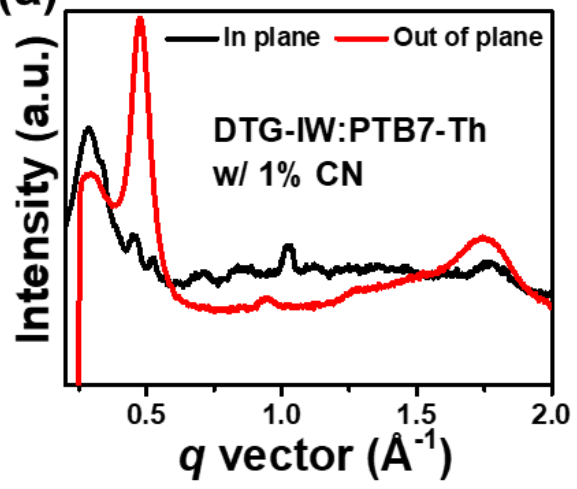

(b)

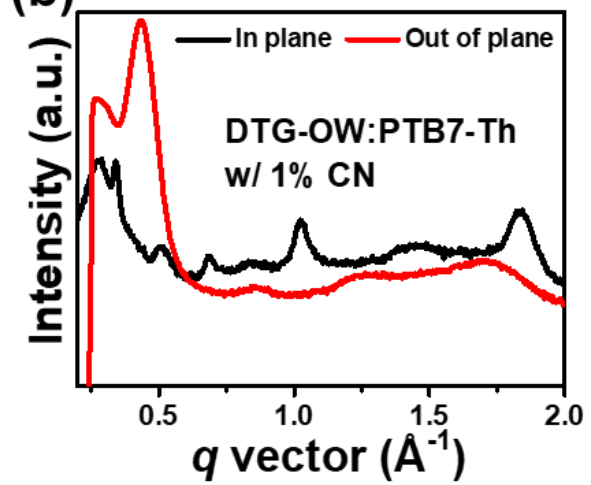

Figure S9. Line cut profiles of optimized (a) DTG-IW:PTB7-Th, (b) DTG-OW:PTB7-Th blend films with $1 \% \mathrm{CN}$.
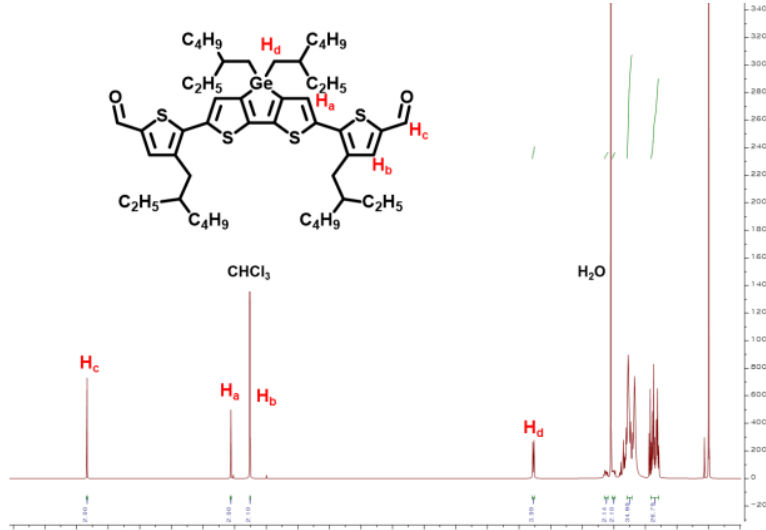
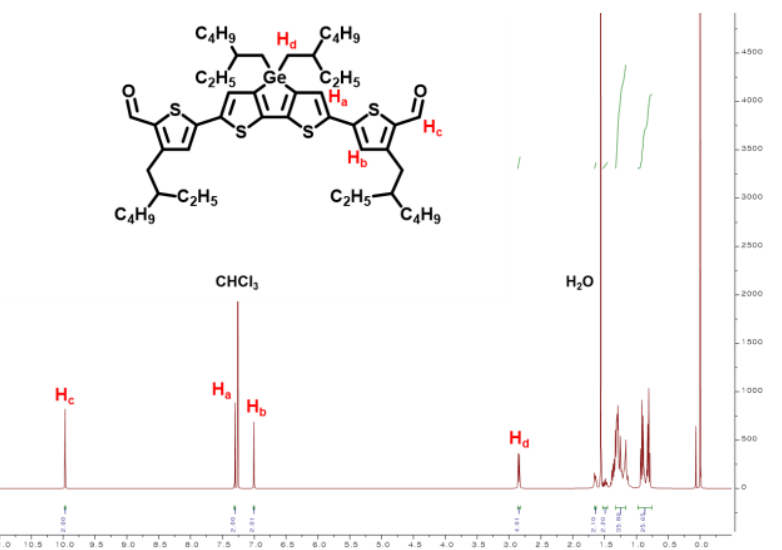

Figure S10. ${ }^{1} \mathrm{H}$ NMR data of compound 4 and 5.
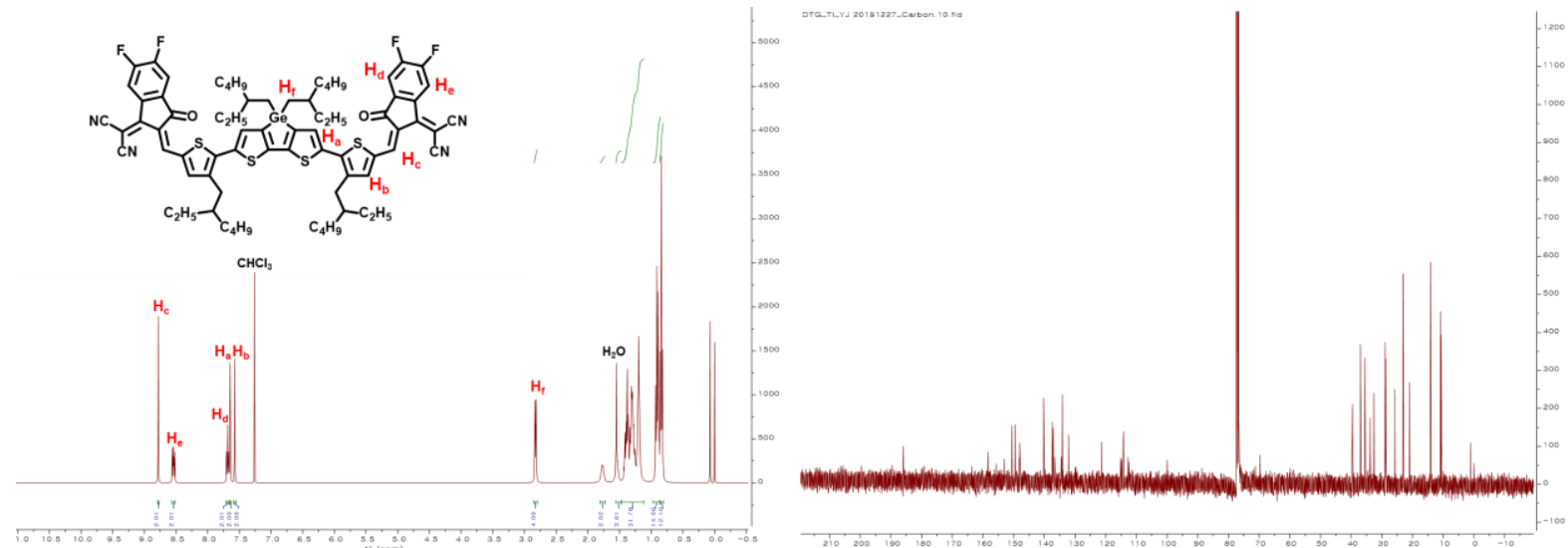

Figure S11. ${ }^{1} \mathrm{H}$ NMR and ${ }^{13} \mathrm{C}$ NMR data of DTG-IW. 


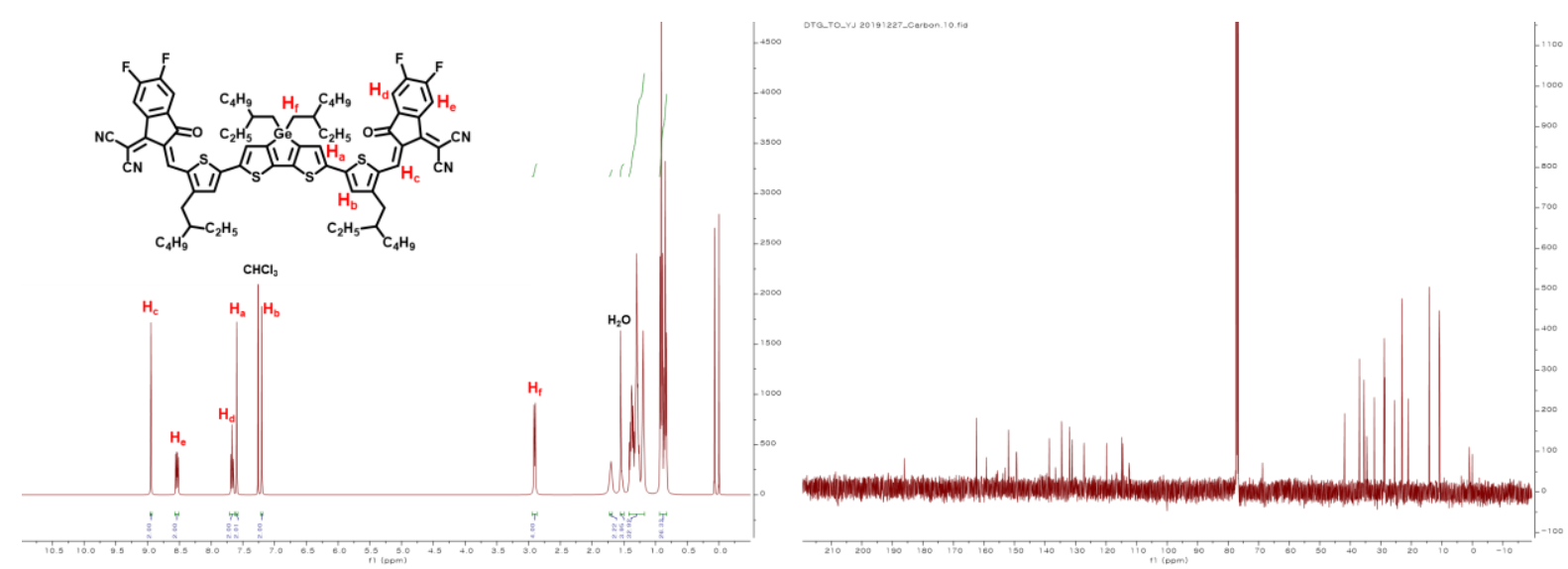

Figure S12. ${ }^{1} \mathrm{H}$ NMR and ${ }^{13} \mathrm{C}$ NMR data of DTG-OW.

\section{REFERENCES}

(1) Fan, B.; Xue, X.; Meng, X.; Sun, X.; Huo, L.; Ma, W.; Sun, Y., High-performance conjugated terpolymer-based organic bulk heterojunction solar cells. J. Mater. Chem. A 2016, 4, 13930-13937.

(2) Liu, S.; Zhou, X.; Zhang, H.; Ou, H.; Lam, J. W. Y.; Liu, Y.; Shi, L.; Ding, D.; Tang, B. Z., Molecular Motion in Aggregates: Manipulating TICT for Boosting Photothermal Theranostics. J. Am. Chem. Soc. 2019, 141, 5359-5368.

(3) Gibson, G. L.; Gao, D.; Jahnke, A. A.; Sun, J.; Tilley, A. J.; Seferos, D. S., Molecular weight and end capping effects on the optoelectronic properties of structurally related 'heavy atom' donor-acceptor polymers. J. Mater. Chem. A 2014, 2, 14468-14480. 